\title{
A Compact XYZ Scanner for Fast Atomic Force Microscopy in Constant Force Contact Mode
}

\author{
Yuen Kuan Yong and S. O. Reza Moheimani
}

\begin{abstract}
The design and characterization of a fast flexurebased, parallel-kinematics XYZ scanner for atomic force microscopy is presented in this article. The objective of the project is to design a AFM scanner with the ability to scan an image at high-speed and high resolution. Finite-element analysis was used to optimize the scanner's design in order to achieve high resonance frequencies. Experimental results show that the scanner has resonance frequencies of $10 \mathrm{kHz}$ at the $X, Y$ and $Z$ axes. To evaluate the performance of the scanner, it was installed into a NT-MDT microscope to obtain images. A $3.5 \mu \mathrm{m} \times 3.5 \mu \mathrm{m}$ image of a calibration grating was successfully recorded up to a scan speed of $625 \mathrm{~Hz}$ in open-loop. Simulations show that the scanner can be operated up to $1025 \mathrm{~Hz}$ with no oscillations observed in the fast axis. The scan rate can be further increased by means of feedback or feedforward control. The device outperforms commercial AFM scanners that typically work at scan frequencies below $20-30 \mathrm{~Hz}$.
\end{abstract}

Index Terms - XYZ scanner, fast atomic force microscopy, flexure, finite-element-analysis

\section{INTRODUCTION}

The Atomic Force Microscope (AFM) is one of the most versatile tools for imaging at extremely high magnification. It has resulted in breakthroughs in areas ranging from bionanotechnology [1] to high-density data storage systems [2]. A typical AFM consists of a cantilever with a sharp probe at one end, a XYZ scanner, a laser and a position sensitive photodiode (PSD). The laser source in the scanning unit is focused at the free end of the cantilever and the PSD captures the laser beam reflected by the cantilever. When operated in constant force contact mode, the cantilever is slowly brought into contact with a sample. The probe-sample interaction causes the cantilever to deflect. This deflection is measured and compared with the preset deflection value. The scanner, on which the sample is placed, is then moved in the Zdirection to reduce the difference between the measured and preset value by means of vertical feedback controls. After the cantilever "lands" on the sample, the scanner is actuated in a zig-zag raster pattern. The vertical feedback controller is used to maintain the cantilever deflection, therefore maintaining the probe-sample interaction force during rastering. A topological image of the sample is constructed from the electrical signal used to drive the Z-axis of the scanner (see Fig. 1).

Constant force contact mode AFM has been widely used to obtain high-resolution images of protein and blood cells

This work was supported by the Australian Research Councils Center of Excellence for Complex Dynamic Systems and Control. The authors are with the School of Electrical Engineering and Computer Science, The University of Newcastle, Callaghan 2308, NSW, Australia. [Yuenkuan.Yong, Reza.Moheimani] @newcastle.edu.au

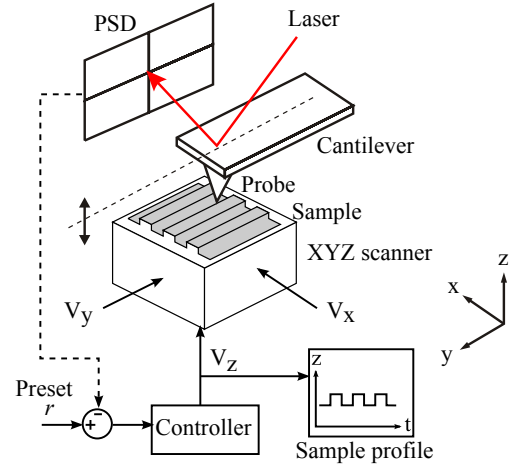

Fig. 1: Schematic of an AFM system in constant force contact mode. $V_{x}, V_{y}$ and $V_{z}$ are the driven signals. $r$ is the preset deflection value of the cantilever.

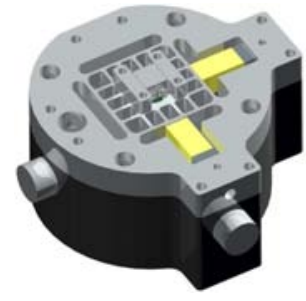

(a)

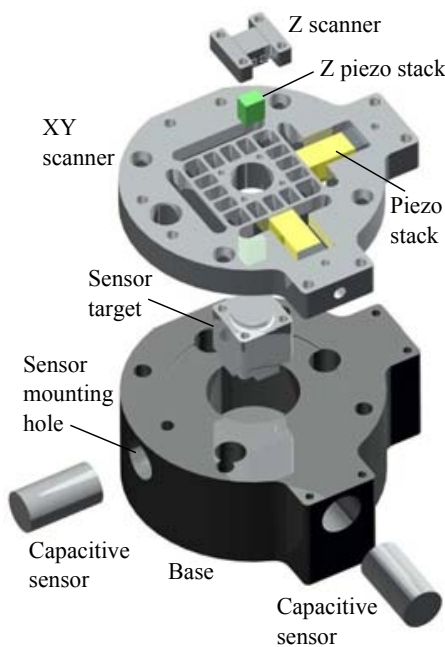

(b)
Fig. 2: CAD drawings of the XYZ scanner. (a) Assembly view. (b) Exploded view.

[1]. The main disadvantage of this mode is its low scan speed. Conventional AFMs typically take a minute or more to record a high resolution image [3]. This clearly inadequate for imaging biological cells that move in milliseconds.

There are many factors limiting the scan speed of an AFM. These factors include: (i) the resonance frequency of the cantilever probe [1], (ii) the resonance frequency of the scanner [4], (iii) the bandwidth of the vertical feedback loop that controls the probe-sample interaction force [1], [5], and (iv) the data acquisition system [4]. 
Piezoelectric tube scanners are the most widely used nanopositioning mechanism in commercial AFMs. They have the lowest resonance frequencies compared to other AFM components such as cantilever probes. A typical piezoelectric tube scanner has a first mechanical resonance frequency of no more than $1 \mathrm{kHz}$. During a raster scan, a triangular waveform is applied to the fast axis and a ramp, or staircase, signal is applied to the slow axis. The triangular waveform contains high-frequency harmonics of the scan frequency. These high-harmonic components tend to excite the resonance frequency of the piezoelectric tube scanner. The low resonant mode of the tube scanner becomes the bottleneck limitation to the AFM speed. Recently, a number of commercial AFMs has appeared in the market that are equipped with flexure-based nanopositioning platforms. Although, overall these platforms have mechanical properties superior to tubes, their scan speeds are similarly limited due to their low first resonance frequencies.

This paper reports the design of a fast parallel-kinematic AFM scanner for high resolution imaging. The main design objectives are to increase the scanner resonance frequencies in $\mathrm{X}, \mathrm{Y}$ and $\mathrm{Z}$ axes and to minimize the cross-coupling effect among the three scan axes. High-speed serial-kinematic scanners have been developed for AFM imaging [1], [6]. While these scanners are suitable for raster scans, they cannot be used for emerging non-raster scan methods, e.g. [7]. A high-speed parallel-kinematic scanner was presented in Ref. [4]. The scanner has high resonant modes, but it contains 9 piezoelectric stack actuators. Each actuator needs to be driven by a dedicated high voltage amplifier. The design reported in [4], is thus more expensive and bulkier. The parallel XYZ scanner presented in this article is much more compact, consisting of only three piezoelectric stack actuators.

This article is organized as follows. In Sec. II, the AFM system and the proposed XYZ scanner are described. A discussion of design considerations and finite-element-analysis of the XYZ scanner are presented in Sec. III. Sec. IV presents the characterization of the scanner by presenting its frequency responses, hysteresis effect and the cross-couplings associated with its three axes. The imaging capability of the scanner is presented in Sec. V.

\section{THE FAST AFM SCANNER}

The proposed AFM scanner consists of a XY nanopositioner, a $\mathrm{Z}$ nanopositioner, a base, three piezoelectric stack actuators, two capacitive sensors and a sensor target. The assembly and exploded views of the scanner are shown in Fig. 2. The scanner is designed to fit into a NT-MDT NTEGRA scanning probe microscope (SPM) shown in Fig. 3. The XYZ scanner is driven by three external voltage amplifiers PiezoDrive PDL200. This amplifier has a gain of 20 and selectable maximum output voltages of $60 \mathrm{~V}, 150 \mathrm{~V}$ and $200 \mathrm{~V}$.

The SPM is configured to operate as an AFM. The AFM's software limits the image resolution relative to scanning speed. At the highest resolution $(256 \times 256$ scan lines $)$ the

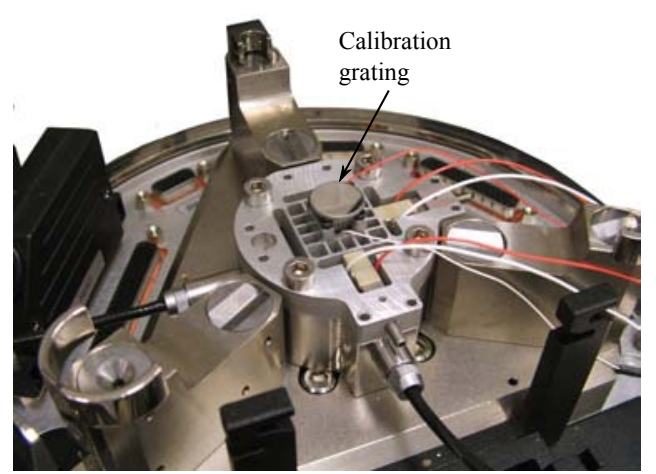

Fig. 3: A NT-MDT NTERGA scanning probe microscope with the original scanner replaced by the flexure-based XYZ scanner.

fastest achievable scanning frequency is limited to $31 \mathrm{~Hz}$. This limits the performance of the XYZ scanner proposed in this work. Therefore, a dSPACE-1103 rapid prototyping system (which has a maximum sampling rate of $80 \mathrm{kHz}$ ) is used to generate the $\mathrm{X}$ and $\mathrm{Y}$ signals and to record the deflection of the cantilever. The $\mathrm{Z}$ signal was controlled using the AFM's software and circuitry. With this setup, the modified AFM can be operated at a scan rate of $625 \mathrm{~Hz}$.

\section{Design Considerations And Finite-element MODELING}

\section{A. Flexure-based design}

The XYZ scanner has a flexible mechanical structure where nanoscale motions are generated through elastic deformation of flexures [8]. With the absence of moving and sliding joints, the scanner provides repeatable and smooth motions without problems such as wear, backlash and friction. This characteristic is highly desirable for nanopositioning devices.

Each motion axis of the XYZ scanner is driven by a piezoelectric stack actuator that is capable of providing high acceleration and large force. For the XY scanner, the central platform (where the $\mathrm{Z}$ scanner is attached) is held by leafspring flexures (see Fig. 4a). The flexures are arranged in such a way so that they are rigid along the direction of motion and are flexible in the axis perpendicular to the motion [9]. As a result, these leaf-spring flexures are used to guide the motion of each axis, and to simultaneously reduce the cross-coupling effect from $\mathrm{X}$-to-Y and vice verse. Other decoupling techniques can be found in Refs. [10], [11].

The $\mathrm{Z}$ scanner consists of two identical sets of leafspring flexures located at both sides of the $\mathrm{Z}$ scanner. A piezoelectric stack actuator is located at the middle of the scanner as shown in Fig. 4b. The leaf-spring flexures are used to only provide vertical motions along the $\mathrm{Z}$ axis. Motions along other axes are restraint by the flexures.

\section{B. Parallel-kinematic structure}

To achieve high-speed scanning, it is required to design a scanner with high resonance frequencies. The $\mathrm{XY}$ and 


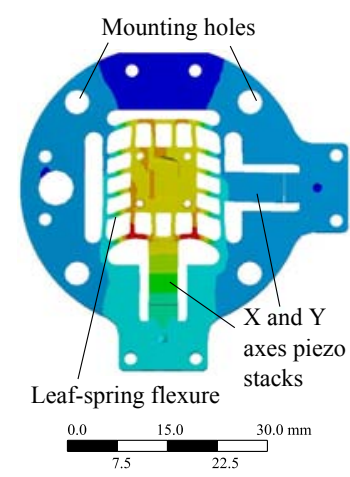

(a)

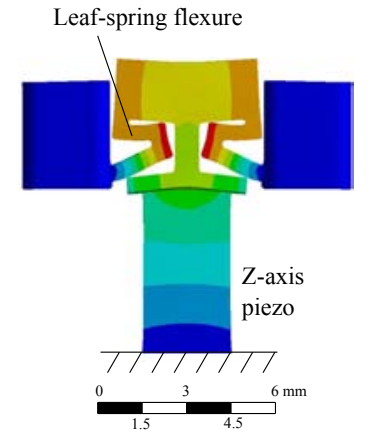

(b)
Fig. 4: Simulated motions and resonance frequencies of the XYZ scanner using ANSYS. (a) The simulation shows that the first resonant mode of the $\mathrm{Y}$ axis appears at $20 \mathrm{kHz}$. The resonant mode of the $\mathrm{X}$ axis is similar. (b) The first resonant mode of the $\mathrm{Z}$ axis appears at $70 \mathrm{kHz}$. The $\mathrm{XY}$ scanner is hidden in the diagram in order to clearly show the motion of the $\mathrm{Z}$ scanner (right) .

$\mathrm{Z}$ scanner described in this article have parallel-kinematic configurations. Parallel structures have high mechanical stiffness, high motion accuracy and high resonance frequencies [12]. These characteristics are suitable for constructing a fast nanopositioner. In addition, the fast axis of a scanner with parallel configuration can be either the $\mathrm{X}$ or the $\mathrm{Y}$ axis due to the similar dynamic properties of the two motion axes.

\section{Material selection}

The resonance frequency of the scanner is also dependent on the material used. Material properties considered to be of great importance are the Young's modulus of elasticity ( $E$ ) and density $(\rho)$. A material with high $E$ to $\rho$ ratio is preferred because a stiff and light material will increase the mechanical stiffness and bandwidth of the scanner.

The motion accuracy of nanopositioning scanners depend on the machining quality of flexures. For example, the flexures of the $\mathrm{Z}$ scanner in Fig. $4 \mathrm{~b}$ have to be as identical as possible to avoid parasitic motions caused by uneven stiffness of flexures. Wire-electrical-discharge-machining (WEDM) is one of the most frequently used machining techniques for fabricating flexures with high precision. WEDM provides cuts with high accuracy and a good surface finish. Materials which have sufficient electrical conductivity to facilitate the WEDM are considered to be highly desirable.

Different materials that satisfy above criteria were considered for the fabrication of the XYZ scanner. Table I compares properties of three candidate materials. Note that all the three materials have sufficient electrical conductivity for WEDM. Titanium alloy has a similar $E / \rho$ ratio compared to Aluminium alloy. However it is more difficult to be machined. Invar alloy has a lower $E / \rho$ value. As a result, Aluminium alloy (A17075) was chosen for fabricating the XYZ scanner.
TABLE I: A comparison of different material properties [13].

\begin{tabular}{ccccc}
\hline Material & $E$ & $\rho$ & $E / \rho$ & Machinability \\
& $\mathrm{GPa}$ & $\mathrm{g} / \mathrm{cm}^{3}$ & & \\
\hline Aluminium alloy (7075) & 72 & 2.81 & 26 & Easy \\
Titanium alloy (Grade 5) & $106-114$ & 4.42 & $24-26$ & Difficult \\
Invar alloy (Invar 36) & 148 & 8.10 & 18.4 & Difficult \\
\hline
\end{tabular}

TABLE II: Specifications of the piezoelectric stack actuators.

\begin{tabular}{cccccc}
\hline $\begin{array}{c}\text { Piezo } \\
\text { stack }\end{array}$ & $\begin{array}{c}\text { Dimension } \\
\mathrm{mm}\end{array}$ & $\begin{array}{c}\text { Disp. } \\
\mu \mathrm{m}\end{array}$ & $\begin{array}{c}\text { Res. freq. } \\
\mathrm{kHz}\end{array}$ & $\begin{array}{c}\text { Capa. } \\
\mathrm{nF}\end{array}$ & $\begin{array}{c}\text { Blocking } \\
\text { Force N }\end{array}$ \\
\hline $\mathrm{X}, \mathrm{Y}$ & $5 \times 5 \times 10$ & $11.8 @ 200 \mathrm{~V}$ & 220 & 380 & 1000 \\
$\mathrm{Z}$ & $3.5 \times 4.5 \times 5$ & $4.6 @ 150 \mathrm{~V}$ & 261 & 90 & 200 \\
\hline
\end{tabular}

\section{Piezoelectric stack actuator}

A piezoelectric stack actuator is constructed by gluing multiple thin layers of piezoelectric ceramics together. Therefore, piezoelectric stack actuators are highly sensitive to tensile (pulling) forces. During high-speed scanning, a piezoelectric stack actuator experiences pushing or pulling forces due to the inertial of its mass and any additional mass it carries. Therefore, preloads must be applied to compensate for the tensile force, and to consequently protecting the piezoelectric stack actuator. For the XYZ scanner proposed in this article, preloads are applied by pressing the piezoelectric stack against the flexures using fasteners. The preload applied to the piezoelectric stack is approximately $100 \mathrm{~N}$.

The electrical aspect of the piezoelectric stack actuator was also taken into consideration to facilitate high-speed operations of the scanner. As reported in [6], a large current is required to drive a piezoelectric stack actuator (which has a large capacitance) at high-speed. This may exceed the current limit of an amplifier. Therefore, the technique proposed in [6] was used to evaluate the feasibility of the chosen piezoelectric stack actuator in this article. The piezoelectric stack actuator that was deemed suitable for the XY scanner is the Noliac SCMAP07 $(5 \times 5 \times 10 \mathrm{~mm}, 380 \mathrm{nF})$. For the $\mathrm{Z}$ scanner, a small Nec-Tokin AE0203D04 piezoelectric stack actuator $(3.5 \times 4.5 \times 5 \mathrm{~mm}, 90 \mathrm{nF})$ is used to minimize weight. Detailed technical specifications of the piezoelectric stack actuators are shown in Table II. The PiezoDrive PDL200 amplifier (which can deliver an average current of $0.1 \mathrm{~A}$ ) was used to drive the piezoelectric stacks. With this setup, it is estimated that the scanner can be driven with a triangular wave (at its full voltage range, i.e. $200 \mathrm{~V}$ ) up to the frequency of $1300 \mathrm{~Hz}$ without exceeding the current limit of the amplifier. The equation used to estimate the maximum operating frequency is $f=0.1 / 200 C$ [14], where $C$ is the load capacitance.

\section{E. Finite-element-analysis of the XYZ scanner}

The dimensions of all flexures were carefully considered in order to maximize the resonance frequencies and to minimize the cross-coupling effect of the scanner. ANSYS was used to conduct a number of design iterations until the desired design criteria was achieved. Constraints were applied to the four mounting holes (see Fig. 4a) to simulate the attachment of the scanner to the base. The finite-element (FE) model 

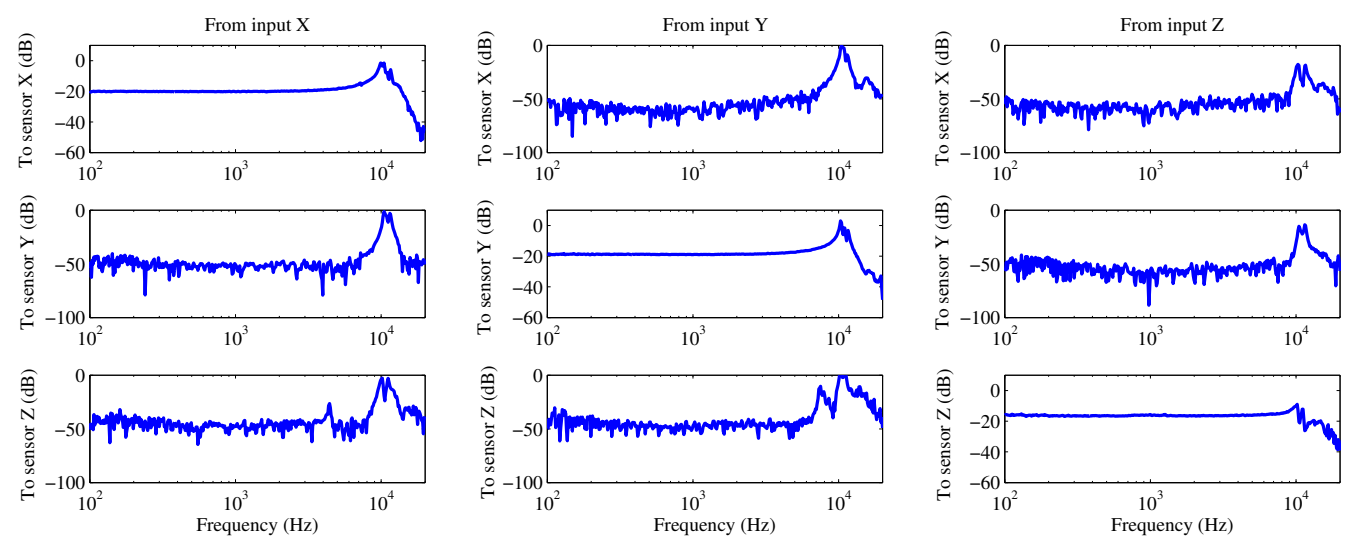

Fig. 5: Frequency responses of the XYZ scanner.

of the scanner are shown in Fig. 4. The first resonant mode of both the $\mathrm{X}$ and $\mathrm{Y}$ axes appears at $20 \mathrm{kHz}$, while the first resonant mode of the $\mathrm{Z}$ axis appears at $70 \mathrm{kHz}$.

\section{CHARACTERIZATION OF THE XYZ SCANNER}

\section{A. Measured frequency responses}

The frequency responses of the scanner were obtained using a HP 35670A dual channel spectrum analyzer. A swept sine input of $100 \mathrm{mVpk}$, with the frequency range of $100 \mathrm{~Hz}$ to $20 \mathrm{kHz}$, was applied to the piezoelectric stack amplifier. The scanner output displacements were measured using capacitive sensors (in volts). The frequency responses of the scanner are plotted in Fig. 5. The first resonance frequency appears at $10 \mathrm{kHz}$ in the $\mathrm{X}, \mathrm{Y}$ and $\mathrm{Z}$ axes, which is different from the FE modeling results. An investigation was carried out to study the differences. It was revealed that the $10 \mathrm{kHz}$ resonant mode is attributed to the base of the scanner which was not simulated in the FE model. A new FE model taking into account the effect of the base was constructed. Simulations show that the two sensor mounting holes at the side of the base (see Fig. 2b) soften the structure, which in turn reduces the resonance frequency of the base significantly. The base design needs further considerations.

\section{B. Hysteresis and cross-coupling measurements}

The hysteretic behavior of piezoelectric stack actuators will often cause artifacts in an open-loop AFM scan. This artifact can be found in the AFM images reported in Sec. $\mathrm{V}$. It is important to characterize the hysteresis effect associated with a scanner, so that a method may be applied to compensate for the hysteresis.

To identify the hysteresis loop, a $100 \mathrm{~Hz}$ triangular waveform was applied to the $\mathrm{X}, \mathrm{Y}$ and $\mathrm{Z}$ axes respectively. A voltage range of $0-200 \mathrm{~V}$ was applied to the $X$ and $Y$ axes, while $0-120 \mathrm{~V}$ was applied to the $\mathrm{Z}$ axis. Table III documents the maximum width of the hysteresis loop and its percentage over the displacement range of each axis. The hysteresis effect can be compensated using techniques such as feedforward control [15], [16]. Accurate hysteresis models such as Preisach model [17] and Prandtl-Ishlinskii operator
TABLE III: Measured hysteresis curves.

\begin{tabular}{cccc}
\hline & Max. width $(\mu \mathrm{m})$ & Disp. range $(\mu \mathrm{m})$ & $\%$ \\
\hline $\mathrm{X}$ & 0.6 & 4.2 & 14 \\
$\mathrm{Y}$ & 0.8 & 5.0 & 16 \\
$\mathrm{Z}$ & 0.5 & 3.4 & 15 \\
\hline
\end{tabular}

[18] can be used in conjunction with feedforward techniques to minimize the hysteresis effect. Besides, charge amplifiers can also be used to linearize piezoelectric stack actuators [8], [19].

The cross-coupling effect among the three axes has been one of the complications that affects the quality of AFM images [20]. Fig. 6 plots the measured cross-coupling motions of the scanner. When a $200 \mathrm{~V}$ peak-to-peak sine-wave was applied to the $\mathrm{X}$ axis, the induced $\mathrm{Y}$ and $\mathrm{Z}$ displacements were $1.58 \%$ and $3.4 \%$ of the $X$ displacement respectively. By applying the sine-wave to the $\mathrm{Y}$ axis, it induces $0.5 \%$ and $2.9 \%$ in the $\mathrm{X}$ and $\mathrm{Z}$ displacements respectively. When the $\mathrm{Z}$ axis is being driven, it induces $0.61 \%$ in the $\mathrm{X}$ displacement and $0.45 \%$ in the $\mathrm{Y}$ displacement. The effect of these cross-coupling motions on AFM images can be reduced by implementing feedback control techniques [11], [20] or by post-processing image data offline [21].

\section{ATOMIC FORCE MICROSCOPY IMAGING}

The scanner was installed in the AFM to obtain images of a MikroMasch TDG01 calibration grating. The grating has parallel ridges with $278 \mathrm{~nm}$ period and approximately $55 \mathrm{~nm}$ height. $3.5 \mu \mathrm{m} \times 3.5 \mu \mathrm{m}$ images of the grating were recorded in constant force contact mode at $10 \mathrm{~Hz}(256 \times 256$ lines), $200 \mathrm{~Hz}(200 \times 200$ lines $)$ and $312.5 \mathrm{~Hz}(128 \times 128$ lines $)$. Due to the bandwidth limitation of the data acquisition system, the scan lines had to be reduced as the scan rate increases. To obtain an image with an acceptable resolution at $625 \mathrm{~Hz}$ (64 $\times 64$ lines), the size of the image had to be reduced to $1.5 \mu \mathrm{m} \times 1.5 \mu \mathrm{m}$.

During a typical raster scan, a triangular waveform is applied to the fast axis and a staircase, or ramp, signal is applied to the slow axis. However, the triangular waveform contains all odd harmonics of the fundamental frequency. 


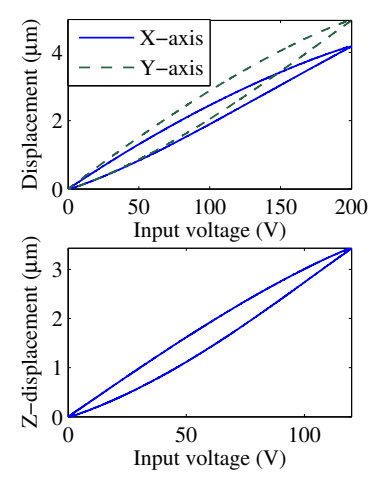

(a)
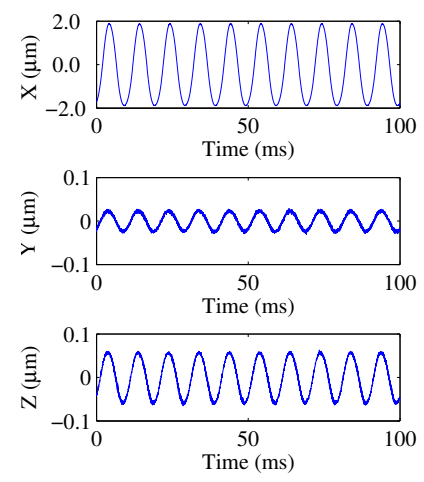
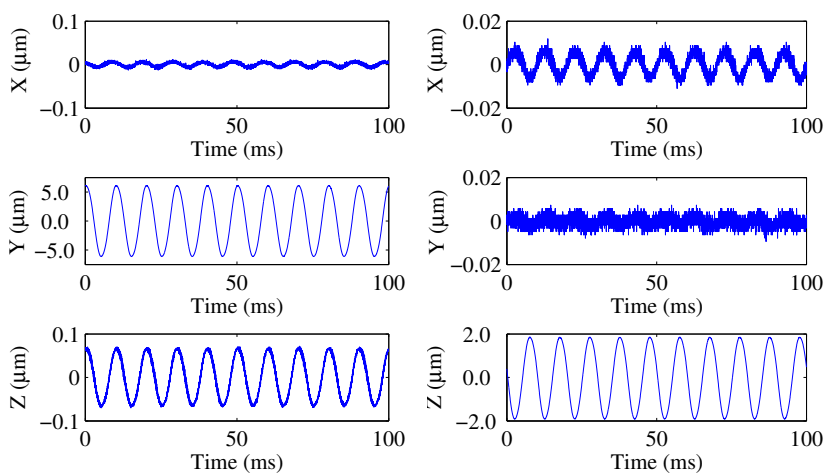

(b)
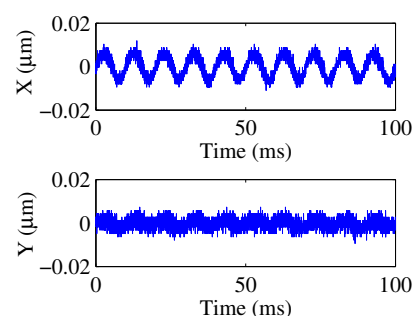

Fig. 6: (a) Hysteresis curves. (b) Cross-coupling measurements at $100 \mathrm{~Hz}$ input sine-wave.

Harmonic components with their frequency contents near the resonance frequency of the scanner will induce vibration in the structure, which in turn introduce artifacts on the scanned images [22].

An optimal signal [23] which has a minimal frequency content above the chosen $K^{\text {th }}$ harmonic was used to drive the fast axis of the scanner during rastering. Fig. 7 shows the simulated scanner output displacements when it is driven by an optimal $(K=7)$ and a triangular signal at $625 \mathrm{~Hz}$ and $1025 \mathrm{~Hz}$, respectively. The optimal input signal improves the scan-induced oscillations at high speeds compared to the triangular signal but produces less range. However, the objective of this work is to achieve fast AFM imaging in constant force contact mode. The optimal signal was deemed suitable to drive the $\mathrm{X}$-axis of the scanner for high-speed scans. Simulations show that an $1025 \mathrm{~Hz}$ optimal signal can be used to drive the $\mathrm{X}$-axis of the scanner without inducing vibrations to the output displacement (see Fig. 7b). However, due to the limitation of our data acquisition system, we were unable to generate an image at this line rate.

Fig. 8 shows the scan images of the grating together with their $\mathrm{X}$ and $\mathrm{Z}$ axis time signals. Images recorded at $200 \mathrm{~Hz}$ and $312.5 \mathrm{~Hz}$ have similar quality as that obtained at $10 \mathrm{~Hz}$. Oscillations were not noticeable in the image and time signal recorded at $625 \mathrm{~Hz}$.

Artifacts caused by the hysteresis effect of the piezoelectric stack actuators were observed in the images where features at the bottom end of the image (2D view) are wider than that at the top end. As discussed in Sec. IV-B, the hysteresis effect can be effectively reduced by feedforward or charge controls.

\section{CONCLUSIONS}

This article presented the design and characterization of a flexure-based XYZ scanner for fast atomic force microscopy. The scanner has resonant peaks at $10 \mathrm{kHz}$ for all three axes. It was installed into a commercial AFM to obtain images. A $3.5 \times 3.5 \mu \mathrm{m}$ area of a calibration grating was scanned

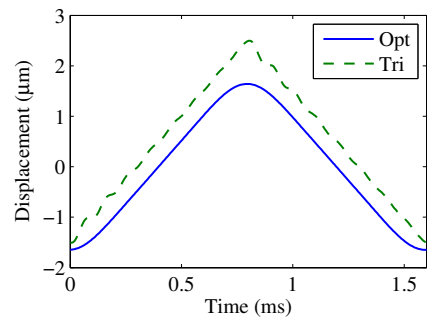

(a)

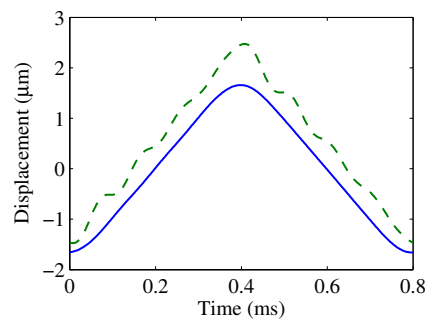

(b)
Fig. 7: Simulated output displacements of the XYZ scanner when driven by an optimal and a triangular input signal respectively at (a) $625 \mathrm{~Hz}$, and (b) $1025 \mathrm{~Hz}$.

at $10 \mathrm{~Hz}, 200 \mathrm{~Hz}, 312.5 \mathrm{~Hz}$ and $625 \mathrm{~Hz}$. Oscillations were not observed in the images and time signals. Simulations show that the scanner can be operated at $1025 \mathrm{~Hz}$ with no scaninduced vibrations observed in the fast axis. However, an image was not recorded at this rate due to the bandwidth limitation of the data acquisition system and the vertical feedback controller. In the future, these two limiting factors will be improved to facilitate high-speed scanning.

FE simulations show that the resonance frequencies of the $\mathrm{X}$ and $\mathrm{Y}$ axes appear at $20 \mathrm{kHz}$ which is different from the experimental results. The $10 \mathrm{kHz}$ resonant peak was arisen from the base which was not properly simulated in the initial FE model. The base will be redesigned in the near future to achieve higher scan rates.

\section{REFERENCES}

[1] T. Ando, T. Uchihashi, and T. Fukuma, "High-speed atomic force microscopy for nano-visualization of dynamic biomolecular processes," Progress in Surface Science, vol. 83, no. 7-9, pp. 337 - 437, 2008.

[2] A. Sebastian, A. Pantazi, S. O. R. Moheimani, H. Pozidis, and E. Eleftheriou, "Achieving sub-nanometer precision in a MEMS storage device during self-servo write process," IEEE Transactions on Nanotechnology, vol. 7, no. 5, pp. 586-595, 2008.

[3] P. K. Hansma, G. Schitter, G. E. Fantner, and C. Prate, "High-speed atomic force microscopy," Science, vol. 314, no. 3799, pp. 601-602, 2006. 


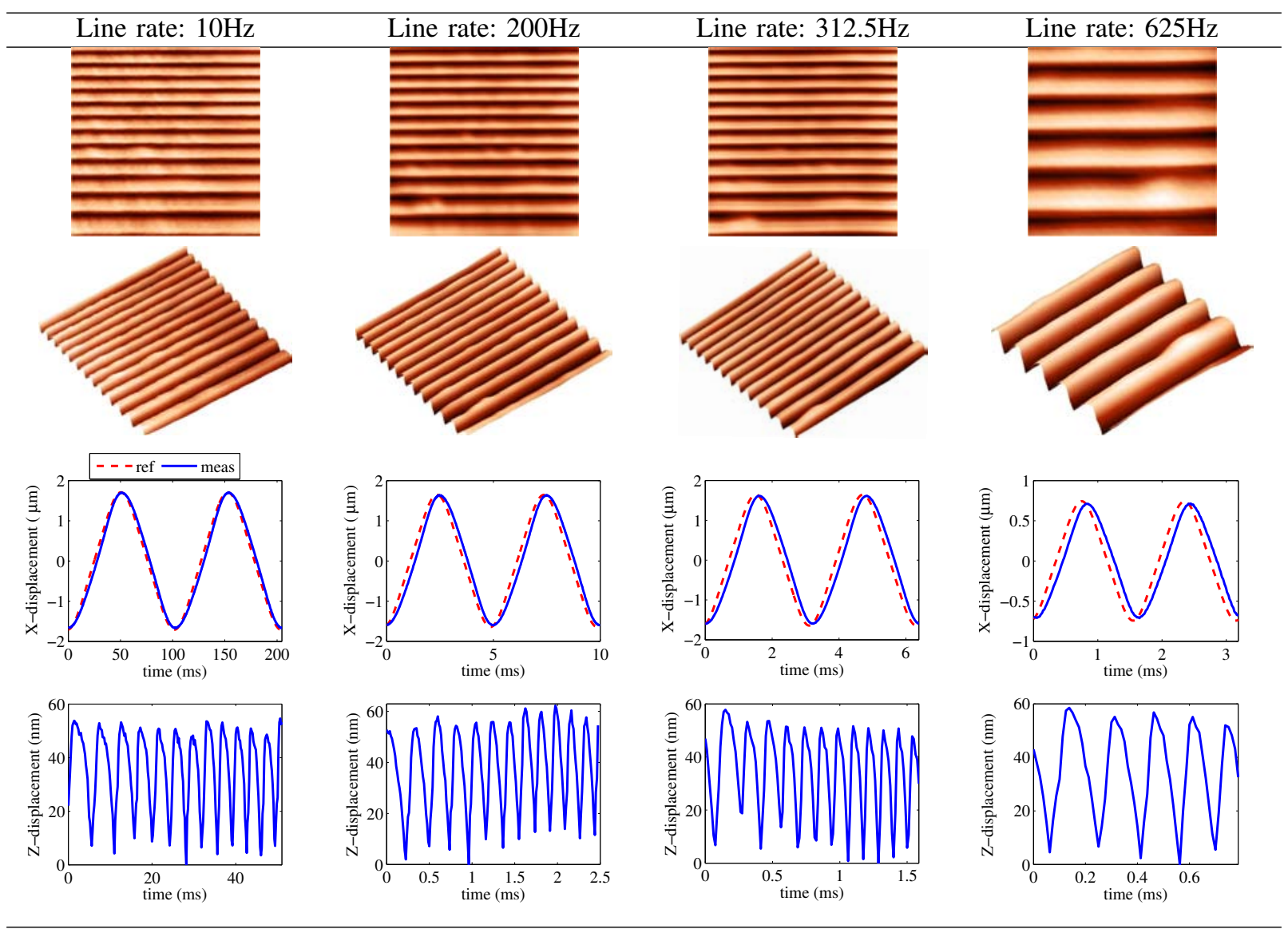

Fig. 8: Scan images of the XYZ scanner in 2D (top) and 3D (middle) together with their time signals (bottom).

[4] G. Schitter, K. J. Åstrom, B. DeMartini, P. J. Thurner, K. L. Turner, and P. K. Hansma, "Design and modeling of a high-speed AFM-scanner," IEEE Trans. Contr. Syst. Tech., vol. 15, no. 5, pp. 906-915, 2007.

[5] A. J. Fleming, "High-speed vertical positioning for contact-mode atomic force microscopy," in Proc. IEEE/ASME International Conference on Advanced Intelligent Mechatronics, Singapore, July 2009, pp. 522-527.

[6] K. K. Leang and A. J. Fleming, "High-speed serial-kinematic AFM scanner: design and drive considerations," Asian Journal of Control, vol. 11, no. 2, pp. 144-153, 2009.

[7] I. A. Mahmood, S. O. R. Moheimani, and B. Bhikkaji, "A new scanning method for fast atomic force microscopy," IEEE Transactions on Nanotechnology, 2010, accepted for publication.

[8] Y. K. Yong, S. Aphale, and S. O. R. Moheimani, "Design, identification and control of a flexure-based XY stage for fast nanoscale positioning," IEEE Trans. Nanotechnology, vol. 8, no. 1, pp. 46-54, 2009.

[9] J. H. Kindt, G. E. Fantner, J. A. Cutroni, and P. K. Hansma, "Rigid design of fast scanning probe microscopes using finite element analysis," Ultramicroscopy, vol. 100, pp. 259-265, 2004.

[10] Y. Li and Q. Xu, "Design and analysis of a totally decoupled flexurebased xy parallel micromanipulator," Robotics, IEEE Transactions on, vol. 25 , no. 3, pp. $645-657$, june 2009.

[11] Y. K. Yong, K. Liu, and S. O. R. Moheimani, "Reducing crosscoupling in a compliant xy nanopositioning stage for fast and accurate raster scanning," IEEE Transactions on Control Systems Technology, 2010, DOI: 10.1109/TCST.2009.2033201 (Accepted for publication).

[12] Y. K. Yong and T.-F. Lu, "The effect of the accuracies of flexure hinge equations on the output compliances of planar micro-motion stages," Mechanism and Machine Theory, vol. 43, pp. 347-363, 2008.

[13] F. Cardarelli, Materials Handbook: A Concise Desktop Reference, 2nd ed. Springer, 2000.

[14] A. J. Fleming, PiezoDrive PDL200 Manual, Newcastle Innovation
Ltd., Industry Development Centre, University Drive, Callaghan NSW 2308, Australia.

[15] S. O. R. Moheimani, "Invited review article: Accurate and fast nanopositioning with piezoelectric tube scanners: Emerging trends and future challenges," Review of Scientific Instruments, vol. 79, no. 7, p. 071101, July 2008, article Number: 071101.

[16] Q. Zou, K. K. Leang, E. Sadoun, M. J. Reed, and S. Devasia, "Control issues in high-speed AFM for biological applications: Collagen imaging example," Asian Journal of Control, vol. 6, no. 2, pp. 164-178, 2004

[17] D. Croft, G. Shedd, and S. Devasia, "Creep, hysteresis, and vibration compensation for. piezoactuators: Atomic force microscopy application," Journal of Dynamic Systems, Measurement, and Control, vol. 123, no. 1, pp. 35-43, 2001.

[18] B. Mokaberi and A. A. G. Requicha, "Compensation of scanner creep and hysteresis for AFM nanomanipulation," IEEE Trans. on Automation Science and Engineering, vol. 5, no. 2, pp. 197-206, April 2008.

[19] A. J. Fleming and S. O. R. Moheimani, "A grounded-load charge amplifier for reducing hysteresis in piezoelectric tube scanners," Review of Scientific Instruments, vol. 76, no. 7, p. 073707, 2005.

[20] G. Schitter and A. Stemmer, "Model-based signal conditioning for high-speed atomic force and friction force microscopy," Microelectronic Engineering, vol. 67-68, pp. 938-944, 2003.

[21] Y. Sun and J. H. L. Pang, "AFM image reconstruction for deformation measurements by digital image correlation," Nanotechnology, vol. 17, pp. 933-939, 2006.

[22] Y. K. Yong, B. Ahmed, and S. O. R. Moheimani, "Atomic force microscopy with a 12-electrode piezoelectric tube scanner," Review of Scientific Instruments, 2010, accepted for publication.

[23] A. J. Fleming and A. G. Wills, "Optimal periodic trajectories for bandlimited systems," IEEE Transactions on Control Systems Technology, vol. 13 , no. 3, pp. 552-562, May 2009. 